siguieron las indicaciones que economistas y financistas hacian con tanta vehemencia.
Por el contrario: incrementaron fuertemenPor el contrario: incrementaron fuertemen-
te su deuda pública para financiar el gasto en Grecia, España, Italia, Estados Unidos Inglaterra, entre otros. El argumento para economias capitalistas habian encontrado un rumbo de estabilidad que aseguraba un rumbo de estabilidad que aseguraba
ingresos tributarios permanentes, no hab razón alguna para no aumentar el gasto salud y pensiones.

Asi, despertamos un día con la sorpresa de que la perfección de los mercados financieros no era tal y la promesa de altos rendimientos era una patraña de unos matemáticos insufribles. La "Gran Moderacion" era un espejismo -por no decir una
mentira- y habia que pagar la deuda, toda ella: privada y pública. En definitiva, la fiesta se habia terminado para todos, inclus nido que rendirse ante la oposición republcana para acordar una reducción de gasto sin precedente, $y$ asegurar asi un poco mas de deuda y no caer en cesación de pagos. Aunque parezca increible: Estados Unidos al borde de la quiebra como cualquier economia del tercer mundo.

thas las bolsas siguen con dias negros y rebotes. Antes fue por los subprime, luego por la deuda europea y ahora por las expectativas de Unidos. Las lecciones han tenido efectos dramáticos y deben traducirse en acciones concretas que cambien la forma de cómo se ha hecho la poltica monetaria, reducominado el debate por masio, que ha politica que considere más seriamente los desequilibrios en los mercados financieros. Además por el lado fiscal, se necesita que los gobiernos ahorren para estabilizar las recesiones; por el contrario tendremos gobiernos débiles con altos déficits que en vez de ayudar en la estabilización, aportarán más a la crisis debido a la falta de dibilidad en los pagos de sus deudas.
LECCIONES PARA CHILE: LAS RECETAS DE MR. KEYNES

portamiento del siste respecto del compuy diferente a sistema financiero no es ses. Un estudio de este autor $y$ de Andrés Sagner indica la posible existencia de un 作 dependiendo de si el ciclo de la economia pasa por momentos expansivos o contractivos, respectivamente.

Asi, en este estudio se observó un aumento de la carteras vencidas en los créditos de consumo despues de periodos de expansión del PIB y /o luego de prolongados y periodos de tasas de interés bajas. Loseso de istas académicos explican este existencia de noticias o creencias sobre el futuro que finalmente no se concretan, con for agentes -consumidores e intererradas que tienen imp- toman decisiones bre el ciclo económico.

Este resultado apoya el argumento de que parte del objetivo de la regulación financiera es controlar la expansión del crédito: las medidas deben ser restrictivas 政 durante una crisis. Asi, la regulación debe ser contracilica y encargarse especialmente de burbujas especulativas cuyo colapso puede danar al sector financiero. Esto, decieras son precedidas de las crisis finanprecios de los activos que emergen después de aperturas financieras e innovaciones y pueden persistir, incluso con la presencia quienes hallan más rentable seguir a una burbuja que ir en contra de ell.

Todo esto es independiente del hecho de que algunos intermediarios financieros (como en el caso La Polar) traspasen límites en la manipulación de los créditos y pago riesgo. Sin duda que la receta es solo ena mano dura para sostener la credibilidad de sistema. Recordemos que una economía de mercado funciona a través de la credibilAstins, codo se puede esfumar. Además, clando aumenta la incertidumpara parar las alzas de la tasa de interes. Mientras no se tenga seguridad de que las dificultades no han terminado, el Banco Central deberia estar preparado para reducciones de tasas y politicas que favorezcan lquidez de los mercados, como las implementadas durante la crisis del 2009. Por otro lado, la politica fiscal debo costablización de conomía y para esto debe esforzarse en mantor un superavit de largo plazo. las

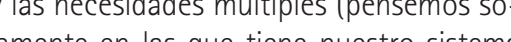
Y fus recursos deben ser aportantes de . En especial si consideramos que el escenaro internacional relevante para la economia chilena se está desacelerando en conunto con un dólar bajo. Es probable que si se materializa este escenario más negativo, nuevamente el gobierno deberá apoyar a la conomia con aumento de gastos.

Mr. Keynes está aún muy vigente con su reencia de que el sector privado alberga en ocasiones conductas irracionales ("espiritus animales"), y por tanto inestabilipar. La leceión parece ser que hace falta rer regulación financiera, más inter-

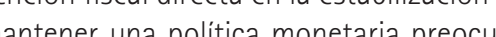
pada también det precio de los pativosy sus burbujas.

John M. Keynes (1883-1946), economista undador de la macroeconomía moderna, nogó por la estabilización macroeconómica, la reducción del desempleo a través de politicas fiscales. Su obra decisiva interés y el dinero (1936), con la que el respuesta a la grave depresión económica desencadenada en todo el mundo a partir do crash de la Bolsa de Nueva York de 1929.

\section{Zanahorias pueden ser garrotes}

Presor Facultad de Economia y Negocios UAH

Si escogiéramos un mantra para la profesión de economista, incentivos deberia son esces los más obvios. Si los recursos vo y un costo de oportinen uso alternaticompensar. Asi por ejemplo qu hay que nas son racionales -se nos dice-, a mayor remuneración, mejor rendimiento; viceversa: si quiero grandes logros necesito proveer de grandes incentivos materiales. ¿Será tan así?

Recientes estudios empiricos en el campo de la economia del comportamiento nos dicen que la racionalidad de los incentivos descansa tambien en elementos no visión más simplista de "g ria-performance”. La investigación en esta materia ha acumulado suficientes estudios novedosos desde que Daniel Kahnema psicólogo de origen, ganara el Nobel de economia en 2002

Una de las investigaciones mas recientes realizada en MIT por Dan Ariely', se propuso indagar en el efecto de los incentives en el desempeno laboral. Lo hizo expe rectivo a trabjer inos in premios por rendimiento. Escogié Indi donde podia pagar bonos sin exceder presupuesto de la investigación.

Los experimentos fueron dos: el primero fue ofrecer tres tipos de bonos por desempeñar correctamente una serie de tare que demandaban cierta habilidad manua mental y creativa. Los bonos ofrecidos me; resertivan, uno alto y uno enlente a un dia un mes $y$ cinco meses. Por cieto todos los participantes rian hacerlo mejor mientras más alta era la recompensa. Sin embargo, y contra lo mo juego. ¿Resultado?

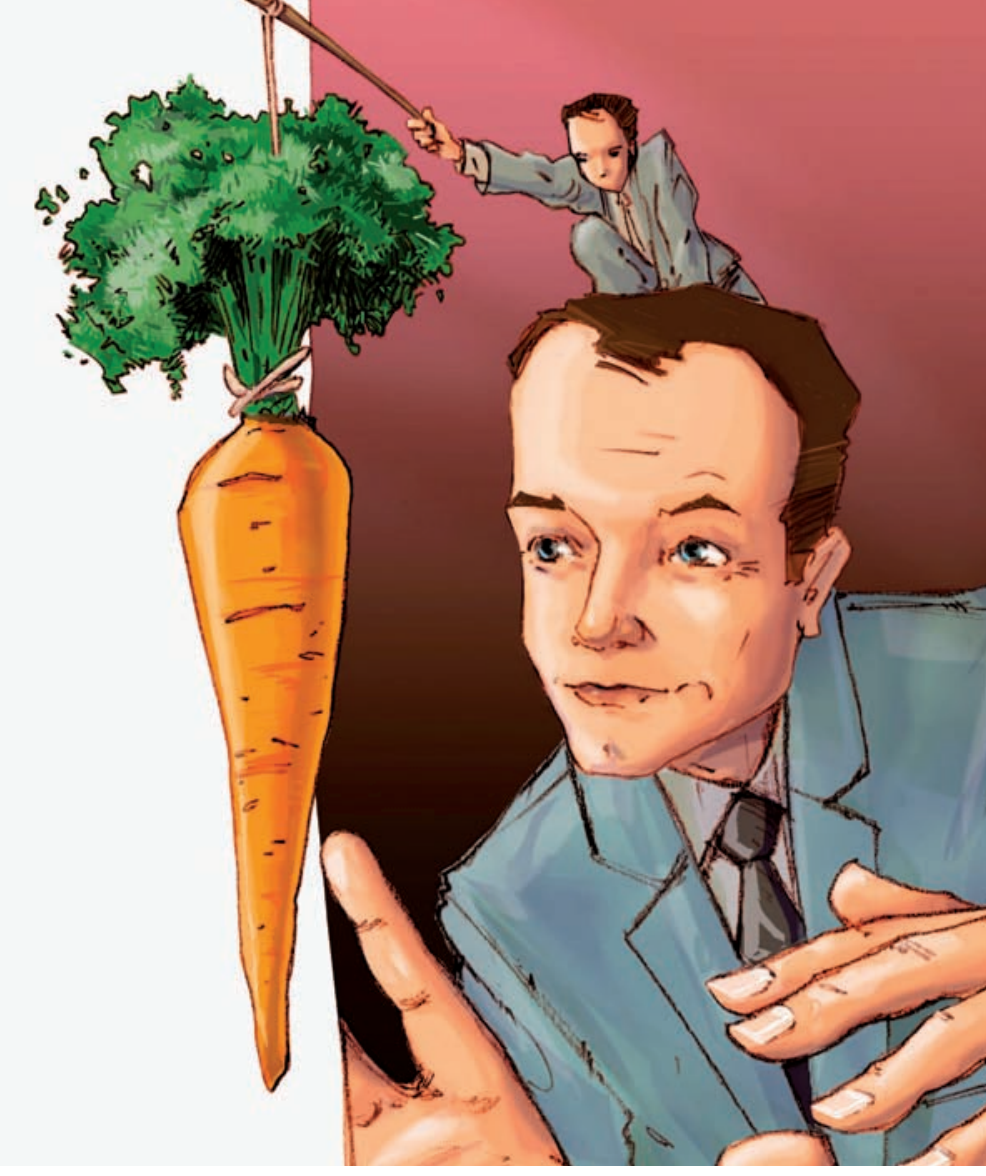

esperado, el grupo que postulaba al bono diseño de esquemas de incentivos en las enorme lo hizo peor. $j$ Por qué? Porque los incentivos contienen un elemento de graficación, pero tambièn uno de estrés. Este ofrecido es demasiado grande por el mis-

mo juego. mismo Ariely con la sugestiva pregunta una alta suma por chistoso si le ofrezco divertida durante los próximos cinco minutos?" Probablemente no. Se estresara pensando en el premio y, si no renuncia al juego, terminara provocando una celebración mas compasiva que genuina.

bonos idénticos a grupos que realizaban separadamente dos tareas distintas. Una ins. Otra, demares mecanicas y rutinaticas y creativas para resolver problemas.

Los bonos funcionaron mejorando de manera directamente proporcional, casi lineal y de acuerdo a lo esperado, dentro de primer grupo. En el segundo en cambio, a recido, baja peros obvo. Alto bono pero ahora derivado de la complejidad de el resultado del bono fue contraproducente. De nuevo la tensión do al deseo de hacerlo bien.

irtamente el estudio podría albergar Fono Facultad: -mail: jrodrigu@uahurtado conomiaynegocios.uahurtado.cl/observatorio Producción OE: :
Periodismo UAH. e la politica pública.

Cuál será, para los accionistas de los sus ejecutivos? escuelas chilenas donde se requierte norme creatividad para sacar de la pread a niños carenciados?

una presunta persona: צ gustaria entrar a un quirófano donde de su tiempo en un bono por operarlo en contra una demanda judicial por ha-

(a) - Y rerponder caso a caso, a de invalidar los incentivos. Si de per(a) antes ni contraproducentes.

RIELY, Dan. The Upside of Irrationality: 作 